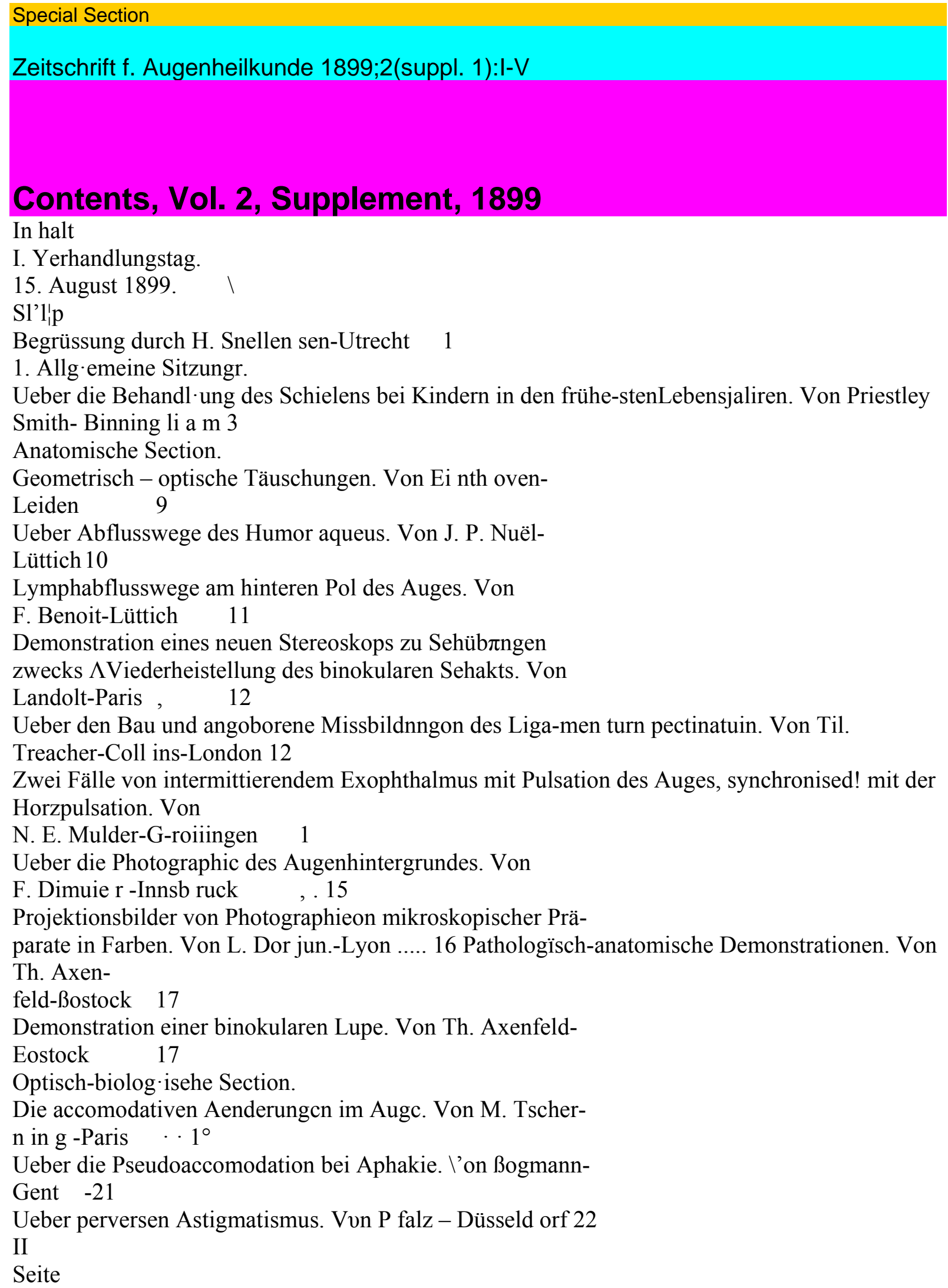


Neues Instrument zur Prüfang der hemianopischen Pupillen-

reaktion. Von Kemp ner- $\Lambda$ Vies baden 23

Ueber Pupillenweite. Aron L. J. Lans-Utrecht . . . 23

Klinisch-therapeutisehe Section.

Ueber benigne postoperative Cyklitis auf infectiöser Basis.

Von 0. Schirmer-Greifswald 25

Zur Klinik der Hyalitis. $\Lambda$ ”on M. Straub-Amsterdam . 26 Ueber Skleritis und ihre Beziehungen zur Myopie. Von

$\Lambda \mathrm{V}$. S ch ön-Leipzig .

Ueber intraokulare Desinfection. Von 0. Haab-Zürich . 28 Zur Behandlung der

Netzhautablösung. Von H. Dor sen.-

Lyon 29

II. YerliancL1·ungstag.

16. August 1899.

2. Allgemeine Sitzung .

Ueber die Ernährungsverhältnisse des Auges. $\Lambda$ ron Th.

Leber-Heidelberg 30

Anatomisehe Section.

Ueber Localisation von Fremdkörpern im Auge mittelst

X-Strahlen. Von K. G r ossmann -Li vor pool ... 33 Ueber freie Metastasenbildung bei

Augengeschwülsten. Von

J. v. Michel- $\Lambda$ Vürzburg 35

Beitrag zur Kenntnis der Arteriosklerose der Augengefässe.

$\Lambda^{\wedge}$ on A. Siegr is t-B asel , 36

Ueber Erkrankung der E, etinalgefässe. Aron 01c Bull-

Christiania 37

Ueber Iritis glaucomatosa. Von $\Lambda$ V. G-oldzieher-Budapest 38 Ueber die Spannung der Retina im Auge. Von Nicolai-

Nijmegen 39

Die drei wesentlichen anatomischen Veränderungen des

Glaukomprozesses. Von $\Lambda V$. Schön-Leipzig . . . . 39

Optisch-biolog $\cdot$ ische Seetion.

Zusammenhang zwischen Accomodation und Convergenz.

Von C. Hess-Marburg .'»

42

Ermüdung infolge von Anstrengung beim binoculären Ein-

fachsehen. Von G·. J. Bull-Paris ... , .... 42 Ueber das Sehen der Schielenden. Von A. Bi els

chow sk y-

Leipzig

Ueber die Elasticität der Sclerotica und ihre Beziehung zur

Entwicklung von Glaucom. $\Lambda$ 'on $\Lambda \mathrm{V}$. Kos ter-Leiden . 45

Klinisch-therapeutisehe Seetion.

Ueber Eisencataracte. $\Lambda$ 7on H. Sattler-Leip zig ... 46

Ueber dift dauernden Erfolge der Myopieoperation. Von

A. v. Hippel-Halle 47

Druok: E. Wertheim, Berlin NW.

in 
Seite

Zur operativen Behandlung des Hornhautkonns. Von

An der son Critc lie t- London $\quad 50$

Ueber Wundschluss bei Hornhautwunden. Von Ernest

Clarke-London $\quad .50$

Die Wirksamkeit der Toxine in der Aetiologie der Bindehaut-

entzündungen. Von Morax-Paris 51

Eine Methode, die diaphane Linse ohne Zerreissung der

Kapsel schnell und vollständig zu trüben. Von Jocqs-

Paris 52

Zur operativen Behandlung des Glaukoms. Von J. Hern-

Semmercote 53

III. Yerliandlungstag.

17. August 1899.

3. Al1/8emeine Sítzung.

Ueber Augenmuskellähmungen auf traumatischer Grundlage.

Von Panas-Paris 54

Anatomisehe Section.

Nervenregenerationen nach Neurectomia optico-ciliaris. Von

Th. Axenfeld-ßo stock 55

Die Keratitis neuroparalytica. Von E. v. Grr ósz -Bu dapest 56

Giebt es im Augeninnern einen primären Krebs oder über-

haupt primäre autonome epitlielialo Neubildungen? Von

E. Krückmann-Leipzig 59

Zur pathologischen Anatomie der Lepra des Auges. Von

E. Franke-Hamburg . . 660

Giebt es eine metastatische Konjunktivitis ? Von F. D. A. C.

van Moll-Rotterdam 60

Ueber Farbenringe, die man normaler $\Lambda$ Veise und bei patho

logischen Zustanden um Lichtquellen heium sehen kann.

Von Druault-Paris 62

Die Atrophie der Opticusnerven bei Tabes dorsalis. Von

E. v. Grósz -Budapest . . . 63

Anatomie des Conus myopicus. Von F. H ein e-Marburg 65

Einiges über die Fasern im Nervus opticus. Von A. Neu-

schüler-Rom 65

Ueber Nervi nervorum des Chiasma. Von L. D o r j un. -Ly on 66

Optisch-biologisehe Section.

Ueber Beeinflussung des centralen Sehens durch seitlicho

Blendung der Netzhaut. Von W. Uhthoff-Breslau . 67 Ueber refraktäre Phasen bei

Augenreflexen. Von H. Zw aar-

demaker uad L. J. Lans-Utrecht 67

Pseudomonochromasie $\Lambda$ \%on Si] ex-Berlin . . . . . 69

Ueber die Periiaetrie der Farben. Von E. S ulzer-Paris 70

Experimentelle Untersuchungen über die periskopischen

Gläser. Von F. Ostwalfc-Paris ... $\quad 70$ 
Seite

Ueber das Maximum der Sehschärfe. Yon P. de 0 barrio

\section{z. Z. Berlin 72}

Wirkung verschiedener Toxine auf die Cornea. Yon H.

Coppez-Brüssel ... 72

Die Schatten beim Skiaskopieren. Yon 0. Neustätter-

München 73

Eine Wandtafel und Modelle zur Yeranschaulichung der Yor-

gänge bei der Skiaskopie. Yon 0. Neu stätter-Münch en 75 Reform der Nummerierung

prismatischer Gläser. Yon E.

Landolt-Paris 75

Kliniseh-therapeutisehe Section.

Die keimtötende Kraft der Thränenflüssigkeit. Yon

Yalude-Paris 76

Behandlung von Hornhautaffectionen mit Injection von Meer-

wasser. Yon P. Dianoux-IS Гantes 77

Therapeutische "Wirkung der Massage des Auges. Yon

Da rier-Paris . . . 77

Neuritis optica in Yerbindung mit Sinuserkrankungen und

Affectionen der Nase. Yon F. de Lapersonne-Lill e . 78 Behandlung der Granulation mit

Elektrolyse. Von H.

C o ppezBrüssel 78

Entfernung des sympathischen Ganglion cervicale superior

bei zwei Fallen von Glaukom und in einem Fall von

Atropbia nervi optici. Yon J. M. Ball-St. Louis . . 79 Zur Behandlung der Cataracta comphcata.

Yon G. G u t -

mann-Berlin $\cdot .81$

Einige seltenere Geschwülste der Augenhöhle. Von H.

Knapp-New- York 81

Eine neue Epicanthusoperation. Yon B. Wichorkiewicz-

Krakau 82

IV. Yerliandlungstag.

18. August 1899.

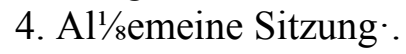

Ueber die Boeinflussung kleineror Fehior der Hornhaut-

krümmung durch operative Massnahmon. Yon C. ßoy-

mond-Turin $8 \mathrm{~B}$

Ueber die Symmetrie unsorer Augen and eine darauf be-

gründete gleichmässige Bezeichnuag der Meridiane. Von

H. Knapp-New York 83

Anatomisehe Section.

Das Listing'sche Gesetz bei Augen mit Muskellähmungen.

Yon K. Grossmann-Liverpool

84

Contrahierter und erschlaffter Ciliarmuskel. Von F. Heine-

Marburg 


\section{Seit $\beta$}

Eine Tafel zur Pruning auf Farbenblindheit mittelst des

Florcontrastes. Yon E. Pflüger-Bern 86

Eine Methode zur Bestimmung der Sehschärfe in der Nähe, für liocligradig Kurzsichtige. Yon E.

Pflüger-Bern . 86

Das Wesen der sogenannten Fuchs'schen Atrophie des Seh-

nerven. Yon R. Grreeff-Berlin

87

Optiseh-biolog $\cdot$ isehe Section.

Ophthalmometrische Untersuchungen über das menschliche Auge nacli dem Tode. Yon S. Hoi

th-Christiania . 87

Die primären Veränderungen bei der bandförmigen Keratitis.

Yon F. Sclnek-Halle 90

Ein neuer stabiler Augenspiegel mit reflex!osem Bilde. Yon

W. Thorner-Berlin . . .90

Kliniseh-therapeutische Section.

Ueber Pannus traclaomatosus und seine Behandlurig mittelst

Periectomie der Cornea. Yon E. Bo eckmann-St. Paul 91 Die Behandlung der

Bindehautentzündungen mit den ver-

scliiedenen Silberverbindungen, insbesondere mit Protargol.

Yon Darier-Paris 92

Die Durchspülung der Thränenwege von den Thränenpunkten aus oline $\mathrm{E}^{1} / 8$ führung der Kanüle in dieselben. Yon 0.

Neustätter-München 93

Die durcl $\alpha$ Krämpfe bewirkten Yeränderungen im Kinder-

auge. Von W. Scliön-Leipzig 94

Behandlung des Nystagmus congenitalis. Yon J. Lavagna-

Monaco

95

Studien über operative Behandlung des Astigmatismus.

Von A. Weber-Darmstadt 96

Schluss-Sitzung. 